\title{
Evaluation of a Foreground Segmentation Algorithm for 3D Camera Sensors
}

\author{
Luca Bianchi, Piercarlo Dondi, Riccardo Gatti, Luca Lombardi, and Paolo Lombardi \\ University of Pavia, Dept. of Computer Engineering and System Science, \\ Via Ferrata 1, 27100 Pavia Italy \\ \{luca.bianchi, piercarlo.dondi, riccardo.gatti, \\ luca.lombardi\}@unipv.it
}

\begin{abstract}
Our interest is focusing on the innovative time-of-flight (TOF) cameras. In this paper we present our approach to foreground segmentation, based on smart-seeded region growing. The seeding strategy makes use of the characteristic intensity signal provided by TOF cameras, and growing is proved by experimental measurements to produce a pixel-wise segmentation of $82 \%-92 \%$ quality. Compared to background subtraction, our approach uses more explicitly the unique capacity of TOF cameras to isolate foreground objects on the basis of their distance. Our work will find an application in gate monitoring and passage surveillance.
\end{abstract}

Keywords: region growing, tracking, gate monitoring, time-of-flight cameras.

\section{Introduction}

The development of time-of-flight (TOF) scanner-less cameras has started a set of new research in computer vision and imaging applications [1]. Realtime grid measurement of depth is now possible using a single compact sensor, whereas traditionally it has been achieved with multi-camera systems.

TOF cameras are active imaging sensors using laser light to measure distances from sensor to scene objects. They are based either on pulsed light or modulated light. In the first case, a coherent wavefront hits the target and high frequency photon gating measures the return time-of-flight. In the latter, the emitted light is modulated and time-of-flight is measured by phase delay detection.

TOF cameras evidently present some advantages. Opposite to laser scanners and active illumination stereo, TOF cameras acquire distance data without employing any moving mechanical part. They can reach video rate, are insensible to shadows, and can measure 3D distance in textured as well as untextured scenes. On the other hand, their measurement range is limited to a few meters and, until today, have a limited resolution. Also, they suffer from noise depending on the amount of incident active light and this causes problems for using multiple TOF cameras. Figure 1 shows the pictures of some TOF cameras currently on the market.

Detection and tracking of people is one application that can benefit from TOF cameras. The third dimension gives trackers more informative measurements and, in principle, this should increase their reliability and precision. Also, 3D sensoring adds 

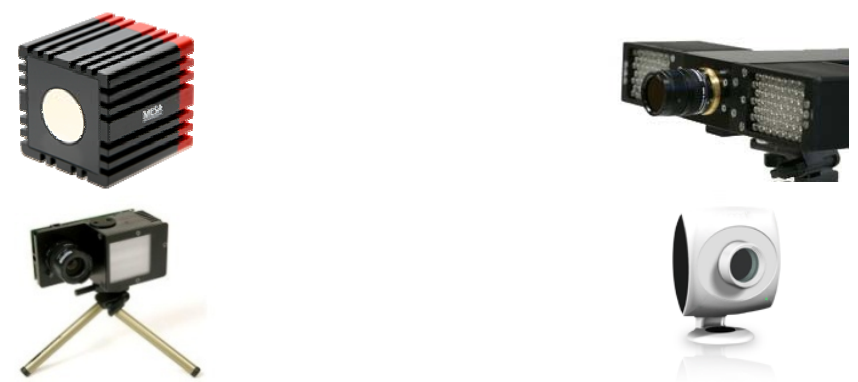

Fig. 1. Different manufacturers provide sensors with diversified aspect ratios and resolutions, as well as sensors based on different physical principles: MESA Imaging SR4000 (top left), PMD[vision] ${ }^{\circledR}$ (top right), and Canesta (bottom left) are phase-based, whereas Zcam by 3DV Systems (bottom right) is based on photon gating

a feature (distance) to help characterizing individual targets and disambiguating between targets in case of mutual occlusions.

The application of TOF cameras to people tracking is limited by the maximum measurement range of these sensors. In contrast with laserscanners - that reach hundreds of meters, TOF cameras have nominal ranges of up to $10 \mathrm{~m}$. The practical range is even shorter, as noise caused by scattering, multi-paths and ambient light severely limits this range to a few meters (2-5m) [10].

We are investigating the potential application of TOF cameras to gate monitoring and passage surveillance. The typical setup would have the camera overlooking the entrance to a room or hall from the ceiling with an angle of $0^{\circ}-45^{\circ}$ from the vertical. Depending on the angle, a camera may capture just heads and shoulders of passers-by from above $\left(0^{\circ}\right)$ or also their facial features if they are facing the camera $\left(>0^{\circ}\right)$. For traditional color-based systems, vertically aligned cameras improve the tracking because people reduce to blobs moving parallel to a 2D plane and occlusions are basically eliminated. However, information on faces and on people's heights is lost unless multiple cameras are used. The vice versa is true if the angle between camera axis and the vertical grows.

The 3D information coming from TOF cameras can help conjugate reliable tracking with face/height detection from a single sensor. In gate monitoring the height of ceiling restricts the operating range within the maximum discussed above. For applications requiring face verification, the near future reserves TOF cameras integrating color and depth pixels (at least two manufacturers have announced or already commercialize such sensors). Instead for the setting with a $0^{\circ}$ angle TOF cameras can determine the height of passers-by and hence distinguish adults from children. This characterization can be of use in some specific scenarios, like when counting resourceful customers that enter or exit a shopping center.

In this paper we present our work on foreground segmentation for TOF images. We propose an approach founded on seed-based region growing that fully exploits the depth keying capability of TOF sensors. Integration of the segmentation algorithm with a Kalman tracker is straightforward, as the tracker naturally provides a set of seeds for the next image. Our system features both components, i.e. region-based segmentation and Kalman filtering, but in this work we disable the tracking algorithm 
with the purpose of evaluating the precision and reliability of segmentation. We report the results obtained on video sequences acquired in a controlled environment and labeled manually by a human operator to define ground truth.

Section 2 presents some previous works related to people detection with TOF cameras. Section 3 describes our foreground segmentation. Section 4 illustrates the experimental results, and Section 5 concludes the paper.

\section{Related Work}

Most TOF-based systems for foreground segmentation up to date have focused on two techniques, namely distance thresholds and background subtraction. The first method sets a "cube of interest" by defining minimum and maximum distances for foreground [2]. All objects falling within the cube are labeled as foreground. If the minimum threshold is set to 0 (camera sensor), the second threshold can be dynamically set after the first object. For our scenario, this approach suffers from problems if the camera angle from the vertical is greater than $0^{\circ}$, for instance $30^{\circ}$. Only one person at a time would be segmented and so a second person partially occluded, would be labeled as background. Furthermore, additional processing is needed because selected pixels are to be clustered into objects.

The second method is inherited by motion detection techniques used in computer vision, notably in video surveillance applications. It consists in creating a model of the object-free scene by means of statistical analysis. The background model is most often pixel-based and only rarely region-based. Then, frame by frame, newly acquired images are compared to the model and pixels which differ significantly are marked as foreground. This technique provides aggregated foreground clusters of objects in motion and includes a noise rejection criterion implicitly in its statistical nature. To our knowledge, it is the most popular approach implemented to date for TOF cameras [4-7], sometimes using both depth and intensity values to build the model.

However, background subtraction suffers from known problems: i) ghosts appearing when background objects leave the scene, ii) absorption of still persons, iii) bootstrapping requiring a few frames, and so on. We propose an approach that more closely exploits the benefits of augmented 3D sensoring and the intrinsic characteristics of the signal acquired by light-modulated TOF cameras.

\section{Segmentation}

We employ the SR3000 in our project, the model previous to the SR4000 shown in Figure 1 in the MESA Imaging line [3]. The SR3000 is a modulated-light camera that we use at $20 \mathrm{MHz}$. Active sources emit in the near infrared (around $850 \mathrm{~nm}$ ) so that no emission is perceivable in the visible spectrum. The SR3000 delivers two maps per frame: the first contains distance information and the other measures the intensity of reflected light (hereafter indicated with D and I, respectively). Figure 2 shows a typical frame sequence taken by the SR3000 device. The images of the first line are the distance maps $\mathrm{D}$, and the images of the second line are the intensity maps I, both at 16 bits. The intensity image depicts the intensity of light reflected by objects in the near 

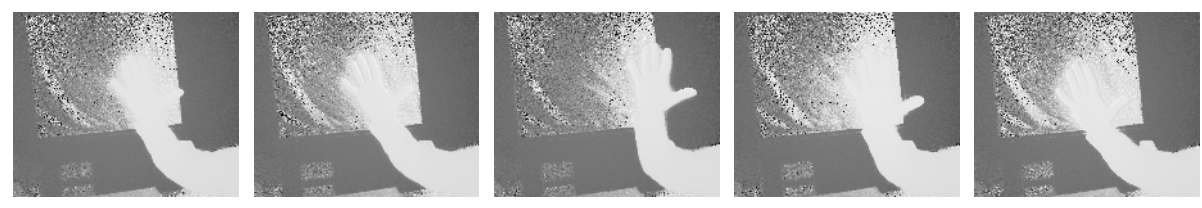

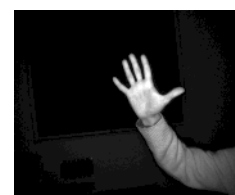

frame 0

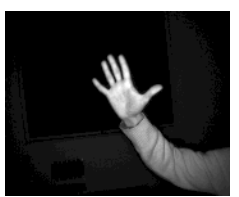

frame 25

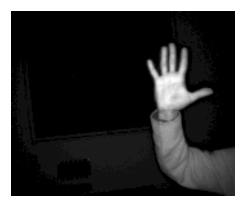

frame 50

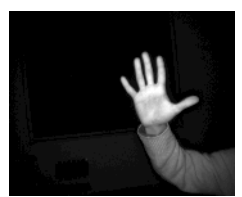

frame 75

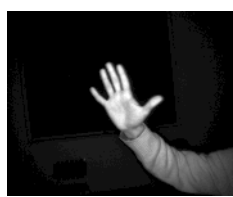

frame 100

Fig. 2. Some frames of one of the sequences analyzed: distance images (top) and intensity images (bottom)

infrared. Almost all of this intensity derives from the internal light sources. Typically, objects near the sensor get more illuminated, while faraway objects receive less light. Hence, peaks in the intensity image tend to correspond to nearby objects. Because in our scenario targets are always closer than the background (the floor or objects shorter than people), we make use of the above observation on the intensity signal to our advantage.

Our approach to segmentation consists in growing regions of foreground objects on the distance map, starting from seeds planted on peaks of the intensity map. The advantages of region growing will be further discussed in Section 5, but briefly they can be summarized in good quality of boundaries (intrinsic noise rejection), independence of background models, and independence of shape models.

To start region growing, we apply a threshold on the intensity image to find some connected components, which then serve as seeds for the distance map D. In practical terms we use peaks of the intensity map I as seeds, under the assumption that the objects to track are the most reflective. In our experiments the threshold will be varied as a parameter to evaluate the performance and robustness of segmentation. We grow a region and then exclude the grown pixels from successive growths. We proceed this way for all seeds in order of descending intensity.

Growing on the distance map has proven experimentally to provide more precise results. Distance data on well illuminated objects (or persons) are homogeneous or smoothly changing (see Figure 2 left). Conversely, growing on the intensity map can be unreliable because its variations are sensible and uncorrelated to object boundaries. For example, folds of the sweater on the arm in Figure 2 (right) reflect light at very different shades.

After experimenting with centroid region growing [8], DBSCAN [9] and other approaches, we have obtained satisfying results with a customized similarity measure. A similarity $\mathrm{S}$ between a cluster pixel $\mathrm{x}$ and a neighboring pixel $\mathrm{y}$ is defined as:

$$
\mathrm{S}(\mathrm{x}, \mathrm{y})=\left|\mu_{\mathrm{x}}-\mathrm{D}_{\mathrm{y}}\right|
$$

In (1), $D_{\mathrm{y}}$ is the distance value of pixel $\mathrm{y}$ and $\mu_{\mathrm{x}}$ is a local parameter related to the mean distance value around $\mathrm{x}$, to be explained soon. The lower is $\mathrm{S}$, the more similar the pixels. In our tests we use 4-connected neighborhoods of radius 1 . 
After defining with $\mathrm{I}_{\mathrm{y}}$ the intensity value of pixel $\mathrm{y}$, and given two constant thresholds $\theta$ and $\lambda$, a pixel $\mathrm{x}$ belonging to a cluster $\mathrm{C}$ absorbs a neighbor $\mathrm{y}$ according to the following predicate:

$$
\left\{\mathrm{x} \in \mathrm{C}, \mathrm{S}(\mathrm{x}, \mathrm{y})<\theta, \mathrm{I}_{\mathrm{y}}>\lambda\right\} \rightarrow\{\mathrm{y} \in \mathrm{C}\}
$$

When a seed is planted, $\mu_{\mathrm{x}}$ in (1) is initialized to $\mathrm{D}_{\mathrm{x}}$. When a neighbor $\mathrm{y}$ of seed $\mathrm{x}$ is absorbed, we compute the average distance value $\mu_{\mathrm{y}}$ in an incremental manner as follows:

$$
\mu_{\mathrm{y}}=\left(\mu_{\mathrm{x}} \cdot \alpha+\mathrm{D}_{\mathrm{y}}\right) /(\alpha+1)
$$

\begin{tabular}{|c|c|c|c|c|}
\hline 10 & 10 & 10 & 11 & 11 \\
\hline 7 & 3 & 8 & 14 & 12 \\
\hline 4 & 5 & 3 & -12 & 12 \\
\hline 4 & 4 & 5 & 12 & 12 \\
\hline 15 & 5 & 16 & 15 & 12 \\
\hline
\end{tabular}

(a)

\begin{tabular}{|c|c|c|c|c|}
\hline 10 & 10 & 10 & 11 & 11 \\
\hline 7 & 3 & $\rightarrow 8$ & 14 & 12 \\
\hline 4 & 5 & 3 & 12 & 12 \\
\hline 4 & 4 & 5 & 12 & 12 \\
\hline 15 & 5 & 16 & 15 & 12 \\
\hline
\end{tabular}

(c)

\begin{tabular}{|c|c|c|c|c|}
\hline 10 & 10 & 10 & 11 & 11 \\
\hline 7 & 3 & 8 & 14 & 12 \\
\hline 4 & 5 & 3 & 12 & 12 \\
\hline 4 & 4 & 5 & -12 & 12 \\
\hline 15 & 5 & 16 & 15 & 12 \\
\hline
\end{tabular}

(b)

\begin{tabular}{|c|c|c|c|c|}
\hline 10 & 10 & 10 & 11 & 11 \\
\hline 7 & 3 & 8 & 14 & 12 \\
\hline 4 & 5 & 3 & 12 & 12 \\
\hline 4 & 4 & 5 & 12 & 12 \\
\hline 15 & 5 & 16 & 15 & 12 \\
\hline
\end{tabular}

(d)

Fig. 3. Starting with a seed in a pixel map (a), the algorithm checks new pixels as shown by the arrows. The example refers to a threshold $\theta=3$ and $\alpha=1$. As pixels are absorbed (b), (c), note that the algorithm never tests twice the same pixel from a given direction. (d) is the final segmentation.

Parameter $\alpha$ is a learning factor of the local mean of D. If pixel y had exactly $\alpha$ neighbors in the cluster, and if the mean of $D$ in these neighbors were exactly $\mu_{x}$, then $\mu_{\mathrm{y}}$ becomes the mean of $\mathrm{D}$ when $\mathrm{y}$ is added to the cluster.

When compared to methods that use global region statistics, like e.g. in centroid region growing, our approach is faster: $\mu_{\mathrm{y}}$ depends only on the history of pixel absorptions until y is first reached by a growing front, and not from later steps. Thus, as soon as a pixel $\mathrm{y}$ is reached by the cluster boundary, it can be tested for absorption. Conversely, in centroid region growing, the addition of a pixel alters the global cluster mean and so the order in which boundary pixels are tested is significant.

Besides, the locality of our approach tolerates greater variations of map values inside a cluster because it produces transitive closures of the similarity S. Both head and shoulders of the same person, lying at slightly different distance from the camera, are more likely to be segmented as the same cluster, rather than two different clusters. 


\section{Experimental Evaluation}

We used a SR3000 TOF camera to test our algorithms. Images are 176x144 pixels and the aperture is $47.5 \times 39.6$ degrees. We have observed an acquisition rate of $20 \mathrm{fps}$ when the camera is in pure acquisition mode, without any further elaboration. When our algorithms are run on a $2.0 \mathrm{GHz}$ Intel Xeon PC, the rate is still high, at $18 \mathrm{fps}$.
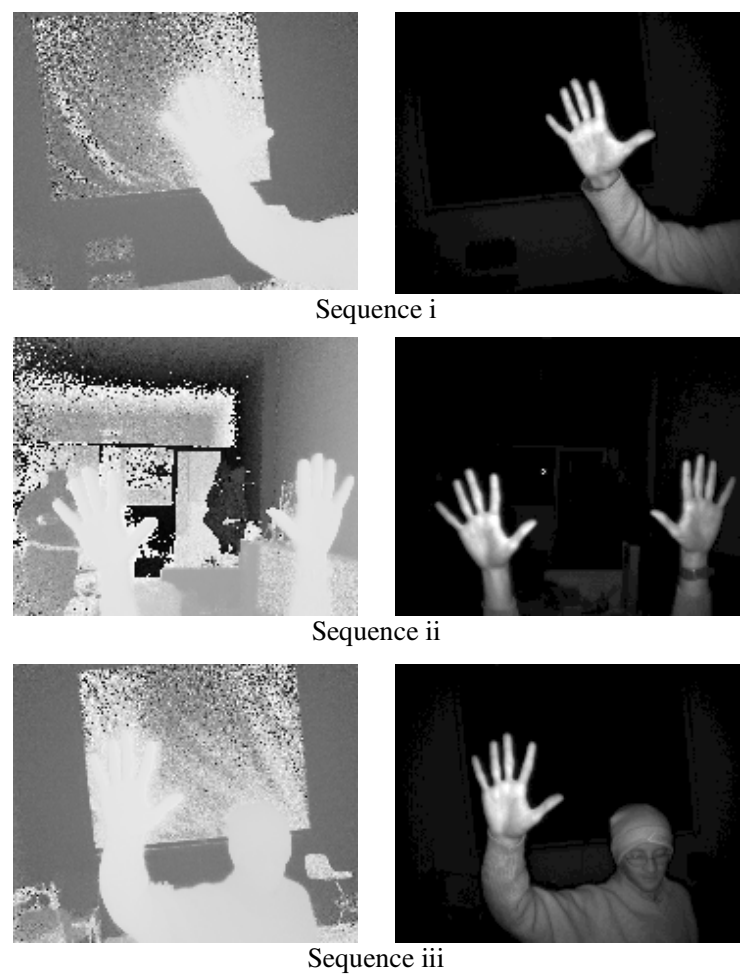

Fig. 4. Distance (left) and intensity (right) of one frame for each of the three sequences used

To assess the performance of our region growing approach, we have manually labeled a set of videos featuring very different conditions: i) a waving hand, ii) two well-separated waving hands, iii) a body and a hand that moves to side (figure 4). The videos have been acquired in different lighting conditions in order to better estimate algorithm performances. Each video consists of 150 frames. The tests have shown that our segmentation algorithm has good performance both with static and moving objects.

As our approach depends upon two parameters, i.e. the similarity threshold $\theta$ and the intensity threshold $\lambda$, we compute the performance for various values of $\{\theta, \lambda\}$. We vary $\theta$ in $[0.5,2] * 2^{10}$ with step $0.125 * 2^{10}$ and $\lambda$ in $[0.5,2] * 10^{3}$ with step $0.1 * 10^{3}$. For each set of parameters, we run the segmentation algorithm on the full sequence and record its performance in terms of the number of pixels correctly labeled as foreground with respect to the ground truth drawn manually. In order to test 
the specific influence of each parameter independently, we run the segmentation algorithm with varying $\theta$ and a fixed $\lambda$, and then with varying $\lambda$ and a fixed $\theta$.

Specifically, using three pixel-wise indices, true positive (TP), false negative (FN) and false positive (FP) - each referred to the number of pixels detected with respect to a manual segmentation taken as ground truth - , we compute the following measures:

- completeness $=\mathrm{TP} /(\mathrm{TP}+\mathrm{FN})$. Completeness is the percentage of the reference data that is explained by the extracted data. The optimal value is 1 .

- correctness $=\mathrm{TP} /(\mathrm{TP}+\mathrm{FP})$. Correctness represents the percentage of correctly extracted foreground data. The optimal value is 1 .

- quality $=\mathrm{TP} /(\mathrm{TP}+\mathrm{FP}+\mathrm{FN})$. Quality is a more general measure combining completeness and correctness into a single measure. The optimal value is 1 .

We compute these values on individual frames, and we take the average on the entire test sequence.

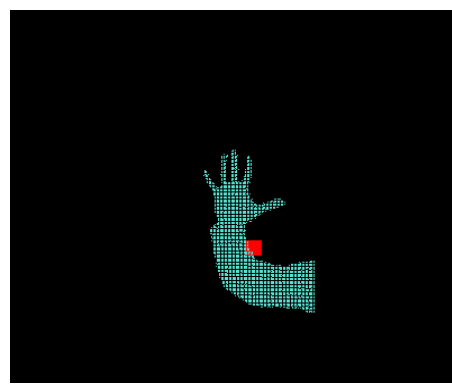

(a)

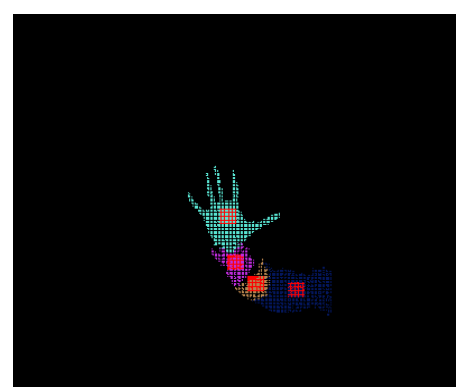

(b)

Fig. 5. An example of segmentation with $\theta$ over (a) and under threshold (b). In the second case the algorithm find four clusters instead of one. The red quads are the centroid of the clusters.

We have observed that generally for a given video there is a threshold over which a further increase in $\theta$ has a negligible contribution, and under which the segmentation failed and the algorithm finds more clusters than those which are really (Figure 6).

On the contrary, a variation of $\lambda$ has always a big impact on the results. Figure 6 shows a clear example of this effect. Observe that for low values of $\lambda$ (Figures $6 a, 6 b$ ) the cluster is more complete but less precise, whereas for high values of $\lambda$ (Figures $6 \mathrm{c}$, 6d) there is an increase in precision (i.e. better correctness) at the expense of loss of pixels (i.e. worse completeness). In this case the best value of $\lambda$ for a good segmentation is near $\lambda=1000$ (b).

The completeness/correctness plot in Figure 7 highlights the impact of $\lambda$. Each dot represents a run with a given value of $\lambda$. All dots referring to the same sequence share the same threshold $\theta$. In fact, given the premises above, for each sequence we have selected a $\theta$ far enough from the threshold that guaranteed target's compactness.

The plot shows that, in optimal conditions (broken and continuous lines), for the larger part tests have scored between $94 \%$ and $97 \%$ in correctness and between $92 \%$ and $96 \%$ in completeness. Those two videos were recorded in a room without sunlight 


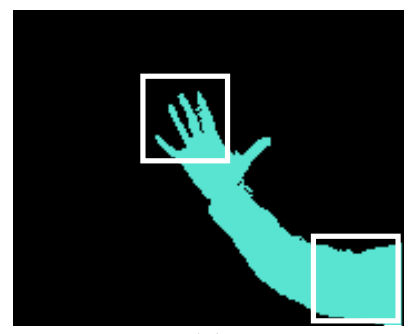

(a)

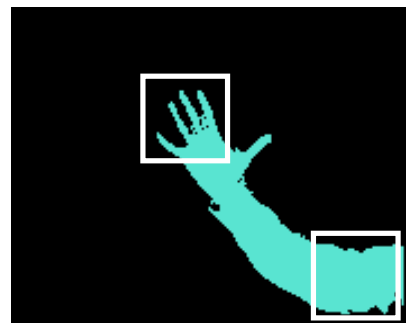

(c)

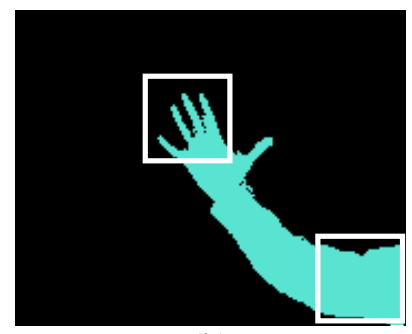

(b)

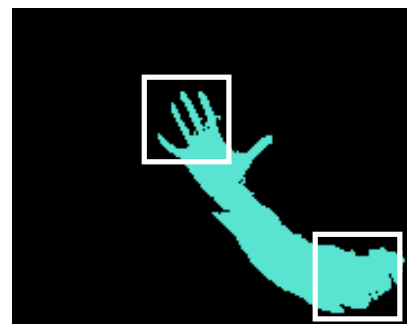

(d)

Fig. 6. The result of our segmentation algorithm applied to one frame of the seq. i with different value of $\lambda$ : (a) is the result with $\lambda=500$, (b) with $\lambda=1000$, (c) with $\lambda=1500$ and (d) with $\lambda=2000$. Notice that, with growing of $\lambda$, the fingers become more precise and the shoulder gradually disappears.

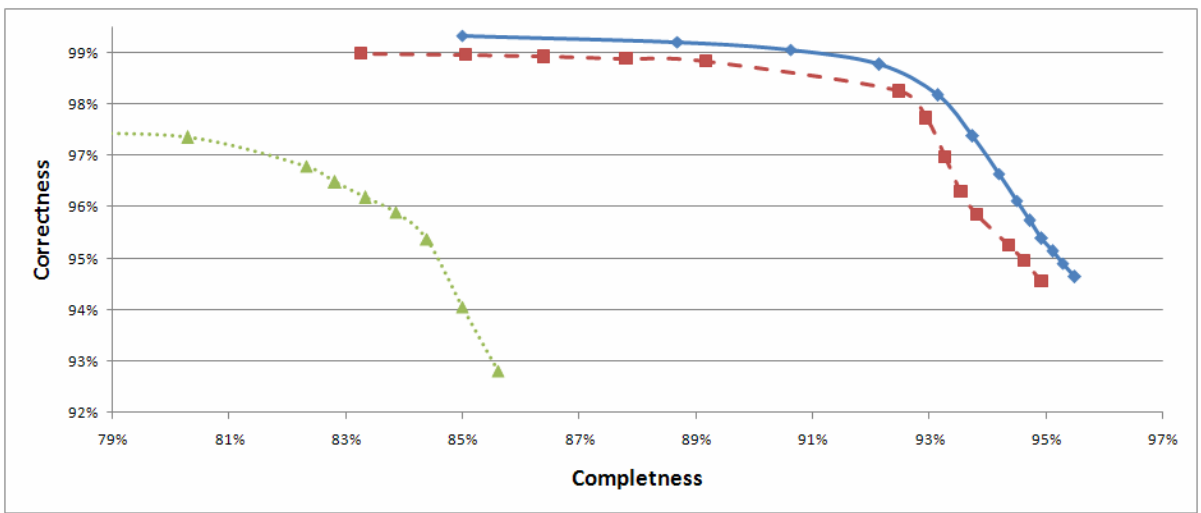

Fig. 7. Correctness/completeness at varying $\lambda$ with a $\theta$ fixed, plot for the three video sequences: red broken line $=$ seq. $\mathrm{i}$; green dotted line $=$ seq. ii; blue continuous line $=$ seq. iii. Every point represents a run of the algorithm throughout the entire sequence with a different parameter set.

to reduce illumination interference. In the worst conditions (dotted line), with much noise generated by sunlight, in spite of a predictable reduction of completeness $(82 \%$ $85 \%$ ), tests have scored high values of correctness between $93 \%$ and $97 \%$, like in the better case. This means that, also in bad condition, the program is able to minimize the number of false positive pixels outside the shape of segmented objects. 
A quantitative measure of goodness for this algorithm is provided by quality. The average quality factor varies between $82 \%$ and $92 \%$.

Although a specific sensitivity study has not yet been done, the graph of Figure 7 suggests that the algorithm is robust enough to parameter changes.

\section{Discussion and Conclusion}

We have presented an approach to foreground segmentation and tracking of objects in video sequences acquired by TOF cameras, envisioning a future application to gate monitoring and passage surveillance. Our approach exploits the intrinsic characteristic of the intensity and distance signals generated by modulated-light TOF to seed a region growing algorithm.

Region growing carries some advantages with respect to other approaches. Our tests on thresholding and histogram-based tracking have confirmed the low noiseresilience inherent to these methods, above all near boundaries because the spatial connection of pixels is neglected. Compared to edge detection (e.g. Canny) region growing guarantees closed regions. As for background subtraction, we use the natural depth-keying feature provided by TOF cameras to eliminate the computational burden of maintaining a background model.

We use a region growing based on cumulative differences rather than on global statistics. Cumulative differences are smoothed by a parameter called neighborhood size, which, for high values, makes the approach similar to a global-statistics approach. The proposed region growing method aggregates regions of smoothly changing distance values, as in the case of head and shoulders or arms and shoulders of the same person. By smart planting of seeds we manage to segment foreground objects with very little processing. Each foreground pixel is visited only once and background pixels are never visited, save for pixels along borders of foreground clusters.

Through testing on sequences manually labeled for ground truth, we have found that the segmentation algorithm presents only a limited sensitivity to the similarity threshold parameter $\theta$. However the intensity threshold parameter $\lambda$ has been found to play a key role. Overall the quality of pixel-wise segmentation ranges from $82 \%$ to $92 \%$ in our tests.

Future work includes the mapping of the segmentation results onto color images coming from webcams, and further refinement of segmentation in the color space.

\section{Acknowledgements}

Financial support for this project was provided by PRIN 2006: "Ambient Intelligence: event analysis, sensor reconfiguration and multimodal interfaces".

\section{References}

1. Kolb, A., Barth, E., Koch, R.: ToF-Sensors: New Dimensions for Realism and Interactivity. In: CVPR 2008, Workshop On Time of Flight Camera based Computer Vision, TOF-CV (2008),

http: / / www-video.eecs.berkeley.edu / Proceedings /CVPR_WS2008/

data/workshops16.htm 
2. Gvili, R., Kaplan, A., Ofek, E., Yahav, G.: Depth Key. In: SPIE Electronic Imaging 2003 Conference, Santa Clara, CA (2003)

3. Oggier, T., Lehmann, M., Kaufmann, R., Schweizer, M., Richter, M., Metzler, P., Lang, G., Lustenberger, F., Blanc, N.: An all-solid-state optical range camera for 3D real-time imaging with sub-centimeter depth resolution (SwissRanger). In: Mazuray, L., Rogers, P.J., Wartmann, R. (eds.) Optical Design and Engineering, Proceedings of the SPIE, vol. 5249, pp. 534-545 (2004)

4. Felder, J., Weiss, S.: Time-of-Flight Imaging for Industrial Applications, Master Thesis, ETH Swiss Federal Institute of Technology Zurich (2007)

5. Witzner, D., Mads, H., Hansen, S., Kirschmeyer, M., Larsen, R., Silvestre, D.: Cluster Tracking with Time-of-Flight Cameras, CVPR 2008, Workshop On Time of Flight Camera based Computer Vision, TOF-CV (2008),

http: / / www-video.eecs.berkeley.edu / Proceedings /CVPR_WS2008/

data/workshops16.htm

6. Guðmundsson, S.A., Larsen, R., Aanæs, H., Pardàs, M., Casas, J.R.: TOF Imaging in Smart Room Environments towards Improved People Tracking. In: CVPR 2008 Workshop On Time of Flight Camera based Computer Vision, TOF-CV (2008), http: / / www-video.eecs. berkeley. edu / Proceedings / CVPR_WS2008/ data/workshops16.htm

7. Bevilacqua, A., Di Stefano, L., Azzari, P.: People tracking using a time-of-flight depth sensor. In: IEEE International Conference on Video and Signal Based Surveillance, pp. 89-94 (2006)

8. Adams, R., Bischof, L.: Seeded Region Growing. IEEE Transactions on Pattern Analysis and Machine Intelligence 16(6), 641-647 (1994)

9. Ester, M., Kriegel, H.P., Sander, J.: A density-based algorithm for discovering clusters in large spatial databases with noise, pp. 226-231. AAAI Press, Menlo Park (1996)

10. Oprisescu, S., Falie, D., Ciuc, M., Buzuloiu, V.: Measurements with ToF Cameras and Their Necessary Corrections. In: Proc. of ISSCS 2007 (2007) 